\title{
Connectivity Analysis of Bipolar Fuzzy Networks
}

\author{
Shu Gong $\mathbb{D}^{1,2}$ Gang Hua, ${ }^{1}$ and Wei Gao ${ }^{3}$ \\ ${ }^{1}$ School of Information and Control Engineering, China University of Mining and Technology, Xuzhou 221116, China \\ ${ }^{2}$ Department of Computer Science, Guangdong University Science and Technology, Dongguan 523083, China \\ ${ }^{3}$ School of Information Science and Technology, Yunnan Normal University, Kunming 650500, China \\ Correspondence should be addressed to Shu Gong; gongshu_gk@126.com
}

Received 15 October 2021; Revised 7 December 2021; Accepted 24 December 2021; Published 19 January 2022

Academic Editor: Wubshet Ibrahim

Copyright (c) 2022 Shu Gong et al. This is an open access article distributed under the Creative Commons Attribution License, which permits unrestricted use, distribution, and reproduction in any medium, provided the original work is properly cited.

\begin{abstract}
Network connectivity is an important factor in data transmission, information sharing, and network defense, and it is a crucial indicator which determines the performance of a network. When a network node or channel contains uncertainties in both positive and negative aspects, the entire network can be modeled with bipolar fuzzy graphs. This paper analyzes the influence of each vertex on the connectivity of the entire network through the definition of the connected state on the bipolar fuzzy graph. The solid results are given, and the new concepts are applied in campus network connectivity analysis. This approach helps to analyze the hidden dangers of the bipolar network, find the weaknesses in the connection, and prevent network attacks in advance.
\end{abstract}

\section{Introduction}

In information science, the connectivity of the network and the connected state of each vertex determine the efficiency of the entire network and the corresponding algorithm. For example, in a protein molecular network, the connected state of the apex determines the molecular activity, which affects the working mechanism of the biological system. In federated learning, the status of each device is not like silos which works all the time. In a certain period of time, devices tend to be in a disconnected state, which leads to the need to select a collection of devices that are in working state during each iteration of federated learning. For various reasons of actual needs, network connectivity has become a hot topic in the field of information technology (see Cheung and Bell [1], Yemini et al. [2], Burla et al. [3], Denison et al. [4], Hummer et al. [5], Ma et al. [6], Gong et al. [7], Gao et al. [8, 9], and Kumar et al. [10]).

Due to the complexity of the network itself, in many application scenarios involving the uncertainty of vertices and edges, it is necessary to borrow tools and methods of fuzzy mathematics. Since the network itself uses vertices to represent sites and edges represent the connecting channels between sites, this network is a graph structure. When it is necessary to describe the uncertain features in the network, the entire model becomes a fuzzy graph model; that is, the membership function of vertices and edges is used to describe this uncertainty in detail. On the other hand, this uncertainty in fuzzy mathematics can often be divided into positive and negative properties. It is well known that the positive membership function cannot reflect the negative uncertainty of objectives. For example, the membership function $\mu_{1}$ stands for "like" and $\mu_{2}$ means "dislike" while $\mu_{1}(x)=0.7$ cannot infer $\mu_{2}(x)=0.3$. A reasonable approach is to use a negative membership function to describe "dislike."

In modeling, the positive uncertainty corresponds to the positive membership function value, and the negative uncertainty corresponds to the negative membership function value. In this setting, the network model becomes a bipolar fuzzy graph, and different types of uncertainties can use different membership functions and ultimately determine various frameworks of the bipolar fuzzy graph. Common ones include bipolar intuitionistic fuzzy graphs, bipolar Pythagorean fuzzy graphs, and so on (see Akram et al. [11], Atef et al. [12], Mahapatra et al. [13], Talebi et al. [14], Gong and Hua [15], Binu et al. [16], Amanathulla et al. [17], Kalathian et al. [18], Akram and Sattar [19], and Mahapatra et al. [20]). 
Very recently, several works contribute to bipolar fuzzy sets and bipolar fuzzy graphs from the perspective of theory and application. Mahmood and Ur Rehman [21] introduced a new notation of bipolar complex fuzzy set which is the fusion of bipolar fuzzy set and complex fuzzy set. Jana [22] studied the multiple attribute group decision-making with bipolar fuzzy numbers in terms of multiple attribute border approximation area comparison approach. Poulik et al. [23] determined the upper and lower boundaries of Randic index of bipolar fuzzy graphs. Ali et al. [24] proposed the theory of an innovative hybrid model called the fuzzy bipolar soft expert sets and applied it to COVID-19 applications. Mehmood et al. [25] raised a new trick to solve LR-type fully bipolar fuzzy linear programming problems with equality restrictions. Ozcelik and Nalkiran [26] introduced an evaluation based on the distance from average solution equipped with trapezoidal bipolar fuzzy sets. Cornejo et al. [27] obtained features of the solvability of bipolar maxproduct fuzzy equations with the standard negation. More related books on bipolar fuzzy graph can be referred to Mathew et al. [28], Pal et al. [29], Akram et al. [30], Chakraverty et al. [31], Chakraverty [32], and Volosencu and Aceves-Fernandez [33].

Binu et al. [34] introduced the connectivity status of vertices in fuzzy graph and classified all the vertices by means of its connectivity status. It inspires us to extend these concepts to more fuzzy settings, and in this work, we focus on bipolar fuzzy setting. The contributions of this work are three-fold: first, we introduce the bipolar connectivity status of each vertex and entire network; then, some theoretical results are inferred in light of fuzzy theory and graph theory; and finally, the given tricks are used in campus network connectivity analysis which aims to prevent network attacks in advance.

The organization of the rest sections are as follows: we present the basic existing concepts on bipolar fuzzy graph in Section 2; new definitions, theoretical results, and proofs are determined in Section 3; a numerical experiment is manifested in Section 4 which simulates university campus network; and finally, the conclusion and remarks are given in the last section.

\section{Definitions in Bipolar Fuzzy Graph Setting}

Let $V$ be a universal set and $A=\left\{\left(v, \mu_{A}^{P}(v), \mu_{A}^{N}(v)\right): v \in V\right\}$ be a bipolar fuzzy set in $V$ with two membership functions $\mu_{A}^{P}: V \longrightarrow[0,1]$ and $\mu_{A}^{N}: V \longrightarrow[-1,0]$. If $B=\left(\mu_{B}^{P}, \mu_{B}^{N}\right)$ is a bipolar fuzzy set in $\widetilde{V}^{2}$ where $\mu_{B}^{P}\left(v, v^{\prime}\right) \leq \min \left\{\mu_{A}^{P}(v), \mu_{A}^{P}\left(v^{\prime}\right)\right\}$,
$\mu_{B}^{N}\left(v, v^{\prime}\right) \geq \max \left\{\mu_{A}^{N}(v), \mu_{A}^{N}\left(v^{\prime}\right)\right\}$ for any $\left(v, v^{\prime}\right) \in \widetilde{V}^{2}$, and $\mu_{B}^{P}\left(v, v^{\prime}\right)=\mu_{B}^{N}\left(v, v^{\prime}\right)=0$ for any $\left(v, v^{\prime}\right) \in \widetilde{V}^{2}-E$, then $G=$ $(V, A, B)$ is a bipolar fuzzy graph (BFG) (the corresponding originally graph is called a crisp graph of fuzzy graph). In what follows, we always use $\wedge$ and $\vee$ instead of minimum and maximum operations, respectively.

Let $G=(V, A, B)$ be a bipolar fuzzy graph. $P=v_{0} v_{1} \ldots v_{k-1} v_{k}$ is a path with length $k$ from $x=v_{0}$ to $y=$ $v_{k}$ in $G$ which is a sequence of different vertices, where $\left(\mu_{B}^{P}\left(v_{i-1}, v_{i}\right)>0, \mu_{B}^{N}\left(v_{i-1}, v_{i}\right)<0\right)$ for $i \in(1, \ldots, k)$. Then,

$\left(\mu_{B}^{P}(x, y)\right)^{k}=\sup \left\{\mu_{B}^{P}\left(x, v_{1}\right) \wedge \mu_{B}^{P}\left(v_{1}, v_{2}\right) \wedge \cdots \wedge \mu_{B}^{P}\left(v_{k-1}, y\right)\right\}$,

and

$$
\left(\mu_{B}^{N}(x, y)\right)^{k}=\inf \left\{\mu_{B}^{N}\left(x, v_{1}\right) \vee \mu_{B}^{N}\left(v_{1}, v_{2}\right) \vee \cdots \vee \mu_{B}^{N}\left(v_{k-1}, y\right)\right\}
$$

Set

$$
\begin{aligned}
& \operatorname{CONN}_{G}^{P}(x, y)=\sup _{k \in \mathbb{N}}\left\{\left(\mu_{B}^{P}(x, y)\right)^{k}\right\}, \\
& \operatorname{CONN}_{G}^{N}(x, y)=\inf _{k \in \mathbb{N}}\left\{\left(\mu_{B}^{N}(x, y)\right)^{k}\right\} .
\end{aligned}
$$

Then, the strength of connectedness between arbitrary $x, y \in V(G)$ in bipolar fuzzy graph $G$ is denoted by

$$
\begin{aligned}
\operatorname{CONN}_{G}(x, y) & =\left(\operatorname{CONN}_{G}^{P}(x, y), \operatorname{CONN}_{G}^{N}(x, y)\right) \\
& =\left(\left(\mu_{B}^{P}(x, y)\right)^{\infty},\left(\mu_{B}^{N}(x, y)\right)^{\infty}\right) .
\end{aligned}
$$

If $\mu_{B}^{P}\left(v, v^{\prime}\right)>0$ and $\mu_{B}^{N}\left(v, v^{\prime}\right)<0$ for any $\left(v, v^{\prime}\right)$ in $G$, then the bipolar fuzzy graph $G$ is connected. If $\mu_{B}^{P}\left(v, v^{\prime}\right)=\min \left\{\mu_{A}^{P}(v), \mu_{A}^{P}\left(v^{\prime}\right)\right\} \quad$ and $\quad \mu_{B}^{N}\left(v, v^{\prime}\right)=\max$ $\left\{\mu_{A}^{N}(v), \mu_{A}^{N}\left(v^{\prime}\right)\right\}$ for any $v, v^{\prime} \in V$, then the bipolar fuzzy graph $G$ is a complete bipolar fuzzy graph.

All edges in bipolar fuzzy graph can be divided into three classes in light of connectedness. An edge $v v^{\prime}$ is strong if $\mu_{B}^{P}\left(v, v^{\prime}\right) \geq \operatorname{CONN}_{G-v v^{\prime}}^{P}\left(v, v^{\prime}\right)$ and $\mu_{B}^{N}\left(v, v^{\prime}\right) \leq \operatorname{CONN}_{G-v v^{\prime}}^{N}$ $\left(v, v^{\prime}\right)$. If $\mu_{B}^{P}\left(v, v^{\prime}\right)>\operatorname{CONN}_{G-v v^{\prime}}^{P}\left(v, v^{\prime}\right)$ and $\mu_{B}^{N}\left(v, v^{\prime}\right)<$ $\mathrm{CONN}_{G-v^{\prime}}^{N}\left(v, v^{\prime}\right)$, then edge $v v^{\prime}$ is called $\alpha$-strong. If $\mu_{B}^{P}\left(v, v^{\prime}\right)=\mathrm{CONN}_{G-v^{\prime}}^{P}\left(v, v^{\prime}\right)$ and $\mu_{B}^{N}\left(v, v^{\prime}\right)=\mathrm{CONN}_{G-v^{\prime}}^{N}$ $\left(v, v^{\prime}\right)$, then the pair $\left(v, v v^{\prime}\right)$ is called $\beta$-strong.

The connectivity index of the bipolar fuzzy graph is defined by Poulik and Ghorai [35], which is formulated by

$$
\begin{aligned}
C I_{B F}(G) & =\left(C I_{B F}^{P}(G), C I_{B F}^{N}(G)\right) \\
& =\left(\sum_{x, y \in V} \mu_{A}^{P}(x) \mu_{A}^{P}(y) \operatorname{CONN}_{G}^{P}(x, y), \sum_{x, y \in V} \mu_{A}^{N}(x) \mu_{A}^{N}(y) \operatorname{CONN}_{G}^{N}(x, y)\right),
\end{aligned}
$$


where $C I_{B F}^{P}(G)$ and $C I_{B F}^{N}(G)$ are positive connectivity index and negative connectivity index of bipolar fuzzy graph $G$.

\section{Connectivity Status in a Bipolar Fuzzy Graph}

The difference between the general frame fuzzy graph and the bipolar fuzzy graph mainly depends on the membership function that describes the fuzzy characteristics of the negative pole contained in the bipolar fuzzy graph. The membership function determines the fuzzy structure characteristics of the negative pole and thus has a decisive influence on the connectivity of the vertices of the entire fuzzy graph. In this section, we introduce the connectivity parameters for bipolar fuzzy graphs.

Definition 1. Let $G=(V, A, B)$ be a bipolar fuzzy graph and $V=\left\{v_{1}, v_{2}, \ldots, v_{n}\right\}$ be the ordered vertex set of $G$. The connectivity status of a vertex $v \in V$ is defined by

$$
\begin{aligned}
C S_{G}(v) & =\left(\operatorname{CS}_{G}^{P}(v), C S_{G}^{N}(v)\right) \\
& =\left(\frac{1}{n-1} \sum_{i=1, v_{i} \neq v}^{n} \operatorname{CONN}_{G}^{P}\left(v_{i}, v\right), \frac{1}{n-1} \sum_{i=1, v_{i} \neq v}^{n} \operatorname{CONN}_{G}^{N}\left(v_{i}, v\right)\right),
\end{aligned}
$$

where $C S_{G}^{P}(v)$ and $C S_{G}^{N}(v)$ are positive connectivity status and negative connectivity status of vertex $v$, respectively. The connectivity status of bipolar fuzzy graph $G$ is denoted by

$$
\begin{aligned}
C S(G) & =\left(C S^{P}(G), C S^{N}(G)\right) \\
& =\left(\frac{1}{n} \sum_{i=1}^{n} C S_{G}^{P}\left(v_{i}\right), \frac{1}{n} \sum_{i=1}^{n} C S_{G}^{N}\left(v_{i}\right)\right),
\end{aligned}
$$

where $C S^{P}(G)$ and $C S^{N}(G)$ are positive connectivity status and negative connectivity status of bipolar fuzzy graph $G$, respectively.

For convenience, we write $C S_{G}(v), C S_{G}^{P}(v)$, and $C S_{G}^{N}(v)$ as $C S(v), C S^{P}(v)$, and $C S^{N}(v)$ for short. The minimum positive connectivity status, maximum positive connectivity status, minimum negative connectivity status, and maximum negative connectivity status of bipolar fuzzy graph $G$ are defined by $\delta_{C S}^{P}(G)=\min _{v \in V(G)}\left\{C S_{G}^{P}(v)\right\}, \quad \Delta_{C S}^{P}(G)=$ $\max _{v \in V(G)}\left\{C S_{G}^{P}(v)\right\}, \quad \delta_{C S}^{N}(G)=\min _{v \in V(G)}\left\{C S_{G}^{N}(v)\right\} \quad$ and $\Delta_{C S}^{N}(G)=\max _{v \in V(G)}\left\{C S_{G}^{N}(v)\right\}$, respectively. A bipolar fuzzy graph is positive connectivity status regular if $\delta_{C S}^{P}(G)=\Delta_{C S}^{P}(G)$ and positive connectivity status regular if $\delta_{C S}^{N}(G)=\Delta_{C S}^{N}(G)$. Two vertices are said to be of positive equistatus (respectively, negative equistatus) in bipolar fuzzy graph $G$ if they have the same positive connectivity status (respectively, negative connectivity status) in G. Two vertices are said to be of equistatus if they are both positive equistatus and negative equistatus.
Example 1. As depicted in Figure 1, $V=\left\{v_{1}, v_{2}, v_{3}, v_{4}, v_{5}\right\}$, $E=\left\{v_{1} v_{2}, v_{2} v_{3}, v_{3} v_{4}, v_{4} v_{5}\right\}, \quad\left(\mu_{A}^{P}\left(v_{i}\right), \mu_{A}^{N}\left(v_{i}\right)\right)=(1,-1)$ for $1 \leq i \leq 5, \mu_{B}^{P}\left(v_{1}, v_{2}\right)=0.6, \mu_{B}^{N}\left(v_{1}, v_{2}\right)=-0.5, \mu_{B}^{P}\left(v_{2}, v_{3}\right)=0.4$, $\mu_{B}^{N}\left(v_{2}, v_{3}\right)=-0.7, \quad \mu_{B}^{P}\left(v_{3}, v_{4}\right)=0.9, \quad \mu_{B}^{N}\left(v_{3}, v_{4}\right)=-0.1$, $\mu_{B}^{P}\left(v_{4}, v_{5}\right)=0.8, \mu_{B}^{N}\left(v_{4}, v_{5}\right)=-0.1, \operatorname{CONN}_{G}^{P}\left(v_{1}, v_{2}\right)=0.6$, $\operatorname{CONN}_{G}^{N}\left(v_{1}, v_{2}\right)=-0.5, \quad \operatorname{CONN}_{G}^{P}\left(v_{1}, v_{3}\right)=0.4, \quad \operatorname{CONN}_{G}^{N}$ $\left(v_{1}, v_{3}\right)=-0.5, \quad \operatorname{CONN}_{G}^{P}\left(v_{1}, v_{4}\right)=0.4, \quad \operatorname{CONN}_{G}^{N}\left(v_{1}, v_{4}\right)=$ $-0.1, \quad \operatorname{CONN}_{G}^{P}\left(v_{1}, v_{5}\right)=0.4, \quad \operatorname{CONN}_{G}^{N}\left(v_{1}, v_{5}\right)=-0.1$, $\operatorname{CONN}_{G}^{P} \quad\left(v_{2}, v_{3}\right)=0.4, \operatorname{CONN}_{G}^{N}\left(v_{2}, v_{3}\right)=-0.7, \operatorname{CONN}_{G}^{P}$ $\left(v_{2}, v_{4}\right)=0.4, \quad \operatorname{CONN}_{G}^{N}\left(v_{2}, v_{4}\right)=-0.1, \quad \operatorname{CONN}_{G}^{P}\left(v_{2}, v_{5}\right)=$ $0.4, \operatorname{CONN}_{G}^{N}\left(v_{2}, v_{5}\right)=-0.1, \operatorname{CONN}_{G}^{P}\left(v_{3}, v_{4}\right)=0.9, \operatorname{CONN}_{G}^{N}$ $\left(v_{3}, v_{4}\right)=-0.1, \quad \operatorname{CONN}_{G}^{P}\left(v_{3}, v_{5}\right)=0.8, \quad \operatorname{CONN}_{G}^{N}\left(v_{3}, v_{5}\right)=$ $-0.1, \quad \operatorname{CONN}_{G}^{P}\left(v_{4}, v_{5}\right)=0.8, \quad \operatorname{CONN}_{G}^{N}\left(v_{4}, v_{5}\right)=-0.1$, $C S_{G}\left(v_{1}\right)=(0.45,-0.3), C S_{G}\left(v_{2}\right)=(0.45,-0.35), C S_{G}\left(v_{3}\right)=$ $(0.625,-0.35), \quad C S_{G}\left(v_{4}\right)=(0.625,-0.1), \quad C S_{G}\left(v_{5}\right)=$ $(0.6,-0.1)$, and $C S(G)=(0.55,-0.24)$.

Furthermore, we infer $\delta_{C S}^{P}(G)=0.45, \Delta_{C S}^{P}(G)=0.625$, $\delta_{C S}^{N}(G)=-0.35$, and $\Delta_{C S}^{N}(G)=-0.1 . v_{1}$ and $v_{2}$ are positive equistatus vertices; $v_{3}$ and $v_{4}$ are positive equistatus vertices; $v_{2}$ and $v_{3}$ are negative equistatus vertices; $v_{4}$ and $v_{5}$ are negative equistatus vertices; however, no two vertices are equistatus.

Our first result reveals the relationship between connectivity index and connectivity status of the bipolar fuzzy graph.

Theorem 1. Let $G=(V, A, B)$ be a bipolar fuzzy graph and $V=\left\{v_{1}, v_{2}, \ldots, v_{n}\right\}$ be the ordered vertex set of $G$. Suppose that $\mu_{A}^{P}(v)=1$ and $\mu_{A}^{N}(v)=-1$ for any $v \in V$. Then,

$$
C I_{B F}(G)=\left(\sum_{i=2}^{n} C S^{P}\left(v_{i}\right)-\sum_{i=2}^{n-1} \sum_{j=i+1}^{n} \operatorname{CONN}_{G}^{P}\left(v_{i}, v_{j}\right), \sum_{i=2}^{n} C S^{N}\left(v_{i}\right)-\sum_{i=2}^{n-1} \sum_{j=i+1}^{n} \operatorname{CONN}_{G}^{N}\left(v_{i}, v_{j}\right)\right) .
$$




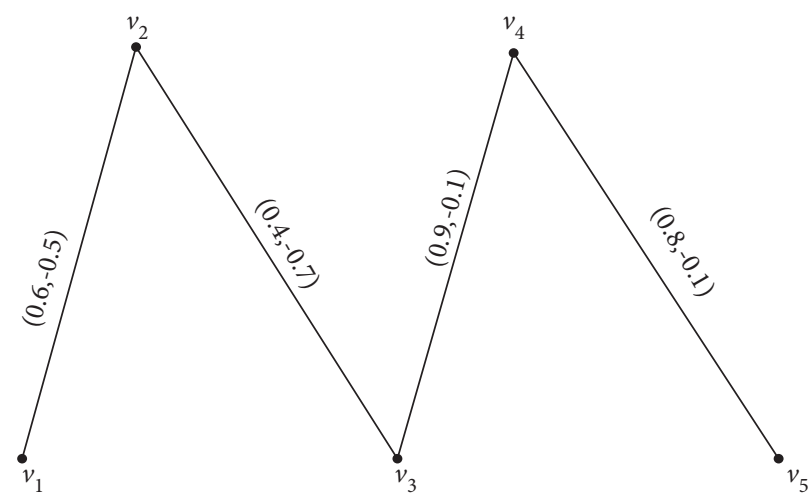

Figure 1: A bipolar fuzzy graph with five vertices.

Proof of Theorem 1. We only prove the negative part, i.e., Under the hypothesis of Theorem 1, we infer

$$
C I_{B F}^{N}(G)=\sum_{i=2}^{n} C S^{N}\left(v_{i}\right)-\sum_{i=2}^{n-1} \sum_{j=i+1}^{n} \operatorname{CONN}_{G}^{N}\left(v_{i}, v_{j}\right) .
$$

$$
\begin{aligned}
C I_{B F}^{N}(G)= & \sum_{x, y \in V} \mu_{A}^{N}(x) \mu_{A}^{N}(y) \operatorname{CONN}_{G}^{N}(x, y) \\
= & \sum_{x, y \in V} \operatorname{CONN}_{G}^{N}(x, y) \\
= & C S^{N}\left(v_{1}\right)+C^{N}\left(v_{2}\right)-\left\{\operatorname{CONN}_{G}^{N}\left(v_{1}, v_{2}\right)\right\}+\operatorname{CS}^{N}\left(v_{3}\right)-\left\{\operatorname{CONN}_{G}^{N}\left(v_{1}, v_{3}\right)+\operatorname{CONN}_{G}^{N}\left(v_{2}, v_{3}\right)\right\} \\
& +\cdots+\operatorname{CS}^{N}\left(v_{n-1}\right)-\left\{\operatorname{CONN}_{G}^{N}\left(v_{n-2}, v_{n-1}\right)+\cdots+\operatorname{CONN}_{G}^{N}\left(v_{1}, v_{n-1}\right)\right\}+\operatorname{CS}^{N}\left(v_{n}\right) \\
& -\left\{\operatorname{CONN}_{G}^{N}\left(v_{n}, v_{n-1}\right)+\cdots+\operatorname{CONN}_{G}^{N}\left(v_{1}, v_{n}\right)\right\} \\
= & \sum_{i=1}^{n} \operatorname{CS}_{G}^{N}\left(v_{i}\right)-\left\{C S_{G}^{N}\left(v_{1}\right)+\operatorname{CONN}_{G}^{N}\left(v_{2}, v_{3}\right)+\cdots+\operatorname{CONN}_{G}^{N}\left(v_{2}, v_{n}\right)\right\} \\
& -\left\{\operatorname{CONN}_{G}^{N}\left(v_{3}, v_{4}\right)+\cdots+\operatorname{CONN}_{G}^{N}\left(v_{3}, v_{n}\right)\right\}-\cdots-\left\{\operatorname{CONN}_{G}^{N}\left(v_{n-2}, v_{n-1}\right)\right. \\
& \left.+\operatorname{CONN}_{G}^{N}\left(v_{n-2}, v_{n}\right)\right\}-\left\{\operatorname{CONN}_{G}^{N}\left(v_{n-1}, v_{n}\right)\right\} \\
= & \sum_{i=2}^{n} \operatorname{CS}^{N}\left(v_{i}\right)-\sum_{i=2}^{n-1} \sum_{j=i+1}^{n} \operatorname{CONN}_{G}^{N}\left(v_{i}, v_{j}\right) .
\end{aligned}
$$

For a complete bipolar fuzzy graph, we have the following statements.

Theorem 2. Let $G=(V, A, B)$ be a bipolar fuzzy graph and $V=\left\{v_{1}, v_{2}, \cdots, v_{n}\right\}$. Assume that $v_{\min }^{P}, v_{\max }^{P}, v_{\min }^{N}, v_{\max }^{N} \in V$ are four vertices (allow some of them to be the same vertex) satisfying $\min _{v \in V(G)}\left\{\mu_{A}^{P}(v)\right\}=\mu_{A}^{P}\left(v_{\min }^{P}\right), \max _{v \in V(G)}\left\{\mu_{A}^{P}(v)\right\}=$
$\mu_{A}^{P}\left(v_{\max }^{P}\right), \min _{v \in V(G)}\left\{\mu_{A}^{N}(v)\right\}=\mu_{A}^{N}\left(v_{\min }^{N}\right)$, and $\max _{v \in V(G)}$ $\left\{\mu_{A}^{N}(v)\right\}=\mu_{A}^{N}\left(v_{\max }^{N}\right)$. Then, the following statements hold:

(1) $\operatorname{CS}^{P}\left(v_{\min }^{P}\right)=\mu_{A}^{P}\left(v_{\min }^{P}\right)$ and $C^{N}\left(v_{\max }^{N}\right)=\mu_{A}^{N}\left(v_{\max }^{N}\right)$

(2) $\delta_{C S}^{P}(G)=C S_{G}^{P}\left(v_{\min }^{P}\right), \quad \Delta_{C S}^{P}(G)=C S_{G}^{P}\left(v_{\max }^{P}\right), \quad \delta_{C S}^{N}$ $(G)=C S_{G}^{N}\left(v_{\min }^{N}\right)$, and $\Delta_{C S}^{N}(G)=C S_{G}^{N}\left(v_{\max }^{N}\right)$

(3) If $\left(\mu_{A}^{P}(v), \mu_{A}^{N}(v)\right)=\left(\mu_{A}^{P}\left(v^{\prime}\right), \mu_{A}^{N}\left(v^{\prime}\right)\right)$, then $v$ and $v^{\prime}$ are equistatus 


\section{Proof of Theorem 2.}

(1) We only verify the negative part. Since $G$ is a complete bipolar fuzzy graph, we have $\mu_{B}^{N}\left(v_{\max }^{N}, v\right)=$ $\mu_{A}^{N}\left(v_{\max }^{N}\right)$ where $v \in V(G)-\left\{v_{\max }^{N}\right\}$, and hence

$$
\begin{aligned}
C^{N}\left(v_{\max }^{N}\right) & =\frac{1}{n-1} \sum_{v \in V(G)-\left\{v_{\max }^{N}\right\}} \mu_{B}^{N}\left(v_{\max }^{N}, v\right) \\
& =\frac{1}{n-1}(n-1) \mu_{A}^{N}\left(v_{\max }^{N}\right) \\
& =\mu_{A}^{N}\left(v_{\max }^{N}\right) .
\end{aligned}
$$

(2) Clearly, $\min _{v \in V(G)}\left\{C S_{G}^{P}(v)\right\}=C S_{G}^{P}\left(v_{\min }^{P}\right), \max _{v \in V(G)}$ $\left\{C S_{G}^{P}(v)\right\}=C S_{G}^{P}\left(v_{\max }^{P}\right), \min _{v \in V(G)}\left\{C S_{G}^{N}(v)\right\}=C S_{G}^{N}$ $\left(v_{\text {min }}^{N}\right)$, and $\max _{v \in V(G)}\left\{C S_{G}^{N}(v)\right\}=C S_{G}^{N}\left(v_{\max }^{N}\right)$. Thus, the results follow from the definition of $\delta_{C S}^{P}(G)$, $\Delta_{C S}^{P}(G), \delta_{C S}^{N}(G)$, and $\Delta_{C S}^{N}(G)$.

(3) It needs to confirm that $v$ and $v^{\prime}$ with the same positive and negative membership function are both positive equistatus and negative equistatus. We only check the correctness of negative equistatus below.

First, we rank all the vertices according to their negative membership function values: $-1 \leq \mu_{A}^{N}\left(v_{n}\right) \leq \mu_{A}^{N}\left(v_{n-1}\right) \leq$ $\cdots \leq \mu_{A}^{N}\left(v_{2}\right) \leq \mu_{A}^{N}\left(v_{1}\right) \leq 0$. Since $\mu_{A}^{N}(v)=\mu_{A}^{N}\left(v^{\prime}\right)$, we suppose $v$ and $v^{\prime}$ are adjacent vertices in the queue. Without loss of generality, assume $\left\{v, v^{\prime}\right\}=\left\{v_{t}, v_{t+1}\right\}$. If $t=1$, then in terms of $v_{\max }^{N}=v_{1}$, we are done by

$$
\begin{aligned}
C S_{G}^{N}\left(v_{2}\right) & =\frac{1}{n-1}\left(\mu_{A}^{N}\left(v_{1}\right)+(n-2) \mu_{A}^{N}\left(v_{2}\right)\right) \\
& =\frac{1}{n-1}\left(\mu_{A}^{N}\left(v_{1}\right)+(n-2) \mu_{A}^{N}\left(v_{1}\right)\right) \\
& =\mu_{A}^{N}\left(v_{1}\right)=C S_{G}^{N}\left(v_{1}\right) .
\end{aligned}
$$

If $t \in\{2, \ldots, n-1\}$, then $\mu_{A}^{N}\left(v_{t}\right)=\mu_{A}^{N}\left(v_{t+1}\right)$ and

$$
\begin{aligned}
C S_{G}^{N}\left(v_{t}\right) & =\frac{1}{n-1}\left(\sum_{i=1}^{t-1} \mu_{A}^{N}\left(v_{i}\right)+(n-t) \mu_{A}^{N}\left(v_{t}\right)\right) \\
& =\frac{1}{n-1}\left(\sum_{i=1}^{t-1} \mu_{A}^{N}\left(v_{i}\right)+\mu_{A}^{N}\left(v_{t}\right)+(n-t-1) \mu_{A}^{N}\left(v_{t}\right)\right) \\
& =\frac{1}{n-1}\left(\sum_{i=1}^{t} \mu_{A}^{N}\left(v_{i}\right)+(n-t-1) \mu_{A}^{N}\left(v_{t+1}\right)\right) \\
& =C S_{G}^{N}\left(v_{t+1}\right) .
\end{aligned}
$$

In all, the Proof of Theorem 2 is finished.

Before introducing the next theorem, we need to extend the concept of saturated fuzzy cycle raised by Mathew et al. [36] to bipolar setting.
Definition 2. Let $G$ be a connected bipolar fuzzy graph. $G$ is $\alpha$-saturated (respectively, $\beta$-saturated) if each vertex is incident at least one $\alpha$-strong edge ( $\beta$-strong edge). $G$ is called saturated if it is both $\alpha$-saturated and $\beta$-saturated. That is to say, at least one $\alpha$-strong edge and one $\beta$-strong edge are incident on every vertex in the bipolar fuzzy graph.

Example 2. As depicted in Figure 2, $V=\left\{v_{1}, v_{2}, v_{3}, v_{4}\right\}$, $E=\left\{v_{1} v_{2}, v_{2} v_{3}, v_{3} v_{4}, v_{4} v_{1}, v_{1} v_{3}\right\}, \quad \mu_{A}^{P}\left(v_{1}\right)=\mu_{A}^{P}\left(v_{2}\right)=\mu_{A}^{P}$ $\left(v_{3}\right)=\quad \mu_{A}^{P}\left(v_{4}\right)=1, \quad \mu_{A}^{N}\left(v_{1}\right)=\mu_{A}^{N}\left(v_{2}\right)=\mu_{A}^{N}\left(v_{3}\right)=\mu_{A}^{N}$ $\left(v_{4}\right)=-1, \mu_{B}^{P}\left(v_{1}, v_{2}\right)=0.6, \mu_{B}^{N}\left(v_{1}, v_{2}\right)=-0.6, \mu_{B}^{P}\left(v_{2}, v_{3}\right)=$ $0.4, \mu_{B}^{N}\left(v_{2}, v_{3}\right)=-0.4, \mu_{B}^{P}\left(v_{3}, v_{4}\right)=0.6, \mu_{B}^{N}\left(v_{3}, v_{4}\right)=-0.6$, $\mu_{B}^{P}\left(v_{4}, v_{1}\right)=0.4, \quad \mu_{B}^{N}\left(v_{4}, v_{1}\right)=-0.4, \quad \mu_{B}^{P}\left(v_{1}, v_{3}\right)=0.2$, and $\mu_{B}^{N}\left(v_{1}, v_{3}\right)=-0.2$.

Clearly, $v_{1} v_{2}$ and $v_{3} v_{4}$ are $\alpha$-strong edges; $v_{2} v_{3}$ and $v_{4} v_{1}$ are $\beta$-strong edges; and $v_{1} v_{3}$ is not strong edge. Hence, $G$ is saturated.

Thereom 3. Let $C=v_{1} v_{2} \ldots v_{n}$ be a saturated fuzzy cycle with $n$ vertices. For $v_{i} \in V(C) i \in\{1, \ldots, n\}$, set $\alpha_{i}^{P}, \beta_{i}^{P}, \alpha_{i}^{N}$, and $\beta_{i}^{N}$ as the positive membership value of $\alpha$-strong edge, positive membership value of $\beta$-strong edge, negative membership value of $\alpha$-strong edge, and negative membership value of $\beta$-strong edge incident to $v_{i}$, respectively. Let $E_{\alpha}$ be the edge of all $\alpha$-strong edges in $C$. Set $u_{\min }^{P} v_{\min }^{P}=\arg \min _{v_{i} v_{i+1} \in E_{\alpha}}\left\{\mu_{B}^{P}\left(v_{i}, v_{i+1}\right)\right\}, \quad u_{\max }^{P} v_{\max }^{P}=$ $\arg \max _{v_{i} v_{i+1} \in E_{\alpha}}\left\{\mu_{B}^{P}\left(v_{i}, v_{i+1}\right)\right\}, \quad u_{\min }^{N} v_{\min }^{N}=\arg \min _{v_{i} v_{i+1} \in E_{\alpha}}$ $\left\{\mu_{B}^{P}\left(v_{i}, v_{i+1}\right)\right\}$, and $u_{\max }^{N} v_{\max }^{N}=\arg \max _{v_{i} v_{i+1} \in E_{\alpha}}\left\{\mu_{B}^{N}\left(v_{i}, v_{i+1}\right)\right\}$ (allow some pair of vertices are the same). Then,

(1) $C S\left(v_{i}\right)=\left(1 / n-1\left(\alpha_{i}^{P}+(n-2) \beta_{i}^{P}\right), 1 / n-1 \quad\left(\alpha_{i}^{N}+\right.\right.$ $\left.\left.(n-2) \beta_{i}^{N}\right)\right), i \in\{1, \ldots, n\}$

(2) If $\alpha$-strong edges of $C$ have equal positive and negative membership values, then all vertices in $C$ are of equistatus

(3) $\delta_{C S}^{P}(G)=C S_{G}^{P}\left(u_{\min }^{P}\right)=C S_{G}^{P}\left(v_{\min }^{P}\right), \Delta_{C S}^{P}(G)=C S_{G}^{P}$ $\left(u_{\max }^{P}\right)=C S_{G}^{P}\left(v_{\max }^{P}\right), \quad \delta_{C S}^{N}(G)=C S_{G}^{N}\left(u_{\min }^{N}\right)=C S_{G}^{N}$ $\left(v_{\min }^{N}\right)$, and $\Delta_{C S}^{N}(G)=C S_{G}^{N}\left(u_{\max }^{N}\right)=C S_{G}^{N}\left(v_{\max }^{N}\right)$

Proof of Theorem 3. Since every vertex in $C$ has degree exactly 2 in $\mathrm{C}$ and for a fixed vertex $v_{i}$ in $\mathrm{C}$, the connectivity of pairs of vertices (contain $v_{i}$ ) is determined by $\beta$-strong edges except one pair with exactly $\alpha$-strong edge. Hence, we can obviously check the statement (1) in view of graph theory.

For statement (2), if the membership value of arbitrary $\alpha$-strong edge is equal, then the statement (1) implies that $\operatorname{CS}^{P}\left(v_{i}\right)$ and $\operatorname{CS}^{N}\left(v_{i}\right)$ are constants for each $v_{i}$, where $i \in\{1, \ldots, n\}$.

Furthermore, the statement (3) is also valid by means of statement (1).

Imitating the vertex classification based on the bipolar connectivity index in Poulik and Ghorai [35], we define the vertex type based on the connectivity status in the following.

Definition 3. Let $G=(V, A, B)$ be a bipolar fuzzy graph and $v \in V$. 


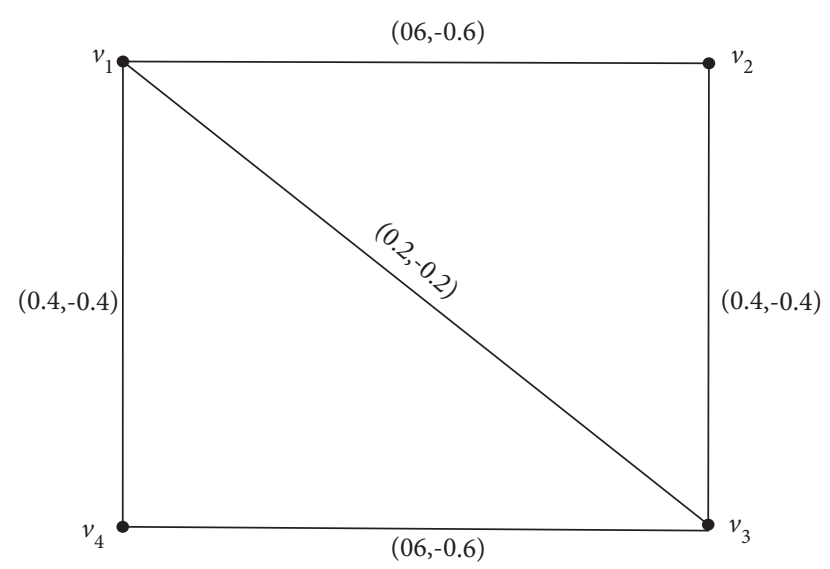

Figure 2: A saturated bipolar fuzzy graph.

(1) If $C S^{P}(G-\{v\})<C S^{P}(G)$ and $C S^{N}(G-\{v\})>$ $C S^{N}(G)$, then $v$ is called a bipolar connectivity status-reducing vertex

(2) If $C S^{P}(G-\{v\})>C S^{P}(G)$ and $C S^{N}(G-\{v\})<$ $C S^{N}(G)$, then $v$ is called a bipolar connectivity status-enhancing vertex

(3) If $C S^{P}(G-\{v\})=C S^{P}(G)$ and $C S^{N}(G-\{v\})=$ $C S^{N}(G)$, then $v$ is called a bipolar connectivity status-neutral vertex

Let $V^{\prime} \subseteq V . V^{\prime}$ is a bipolar connectivity status-reducing vertex set if all its vertices are bipolar connectivity statusreducing vertex; $V^{\prime}$ is a bipolar connectivity status enhancing vertex set if all its vertices are bipolar connectivity status-enhancing vertex; and $V^{\prime}$ is a bipolar connectivity status-neutral vertex set if all its vertices are bipolar connectivity status-neutral vertex.

Example 3. As depicted in Figure 3, $V=\left\{v_{1}, v_{2}, v_{3}, v_{4}, v_{5}\right\}$, $E=\left\{v_{1} v_{2}, v_{2} v_{3}, v_{3} v_{4}, v_{4} v_{1}, v_{2} v_{5}, v_{3} v_{5}, v_{4} v_{5}\right\}, \quad \mu_{A}^{P}\left(v_{1}\right)=\mu_{A}^{P}$ $\left(v_{2}\right)=\mu_{A}^{P}\left(v_{3}\right)=\mu_{A}^{P}\left(v_{4}\right)=\mu_{A}^{P}\left(v_{5}\right)=1, \quad \mu_{A}^{N}\left(v_{1}\right)=\mu_{A}^{N}\left(v_{2}\right)=$ $\mu_{A}^{N}\left(v_{3}\right)=\mu_{A}^{N}\left(v_{4}\right)=\mu_{A}^{N}\left(v_{5}\right)=-1, \quad \mu_{B}^{P}\left(v_{1}, v_{2}\right)=0.5, \quad \mu_{B}^{N}$ $\left(v_{1}, v_{2}\right)=-0.5, \mu_{B}^{P}\left(v_{2}, v_{3}\right)=0.3, \mu_{B}^{N}\left(v_{2}, v_{3}\right)=-0.8, \mu_{B}^{P}\left(v_{3}\right.$, $\left.v_{4}\right)=0.8, \mu_{B}^{N}\left(v_{3}, v_{4}\right)=-0.3, \mu_{B}^{P}\left(v_{4}, v_{1}\right)=0.4, \mu_{B}^{N}\left(v_{4}, v_{1}\right)=$ $-0.5, \mu_{B}^{P}\left(v_{2}, v_{5}\right)=0.2, \mu_{B}^{N}\left(v_{2}, v_{5}\right)=-0.9, \mu_{B}^{P}\left(v_{3}, v_{5}\right)=0.8$, $\mu_{B}^{N}\left(v_{3}, v_{5}\right)=-0.1, \quad \mu_{B}^{P}\left(v_{4}, v_{5}\right)=0.1, \quad \mu_{B}^{N}\left(v_{4}, v_{5}\right)=-0.9$, $\operatorname{CONN}_{G}^{P}\left(v_{1}, v_{2}\right)=0.5, \operatorname{CONN}_{G}^{N}\left(v_{1}, v_{2}\right)=-0.5, \operatorname{CONN}_{G}^{P}$ $\left(v_{1}, v_{3}\right)=0.4, \operatorname{CONN}_{G}^{N}\left(v_{1}, v_{3}\right)=-0.5, \quad \operatorname{CONN}_{G}^{P}\left(v_{1}, v_{4}\right)=$ $0.4, \mathrm{CONN}_{G}^{N}\left(v_{1}, v_{4}\right)=-0.5, \mathrm{CONN}_{G}^{P}\left(v_{1}, v_{5}\right)=0.4, \mathrm{CONN}_{G}^{N}$ $\left(v_{1}, v_{5}\right)=-0.5, \quad \operatorname{CONN}_{G}^{P}\left(v_{2}, v_{3}\right)=0.4, \quad \operatorname{CONN}_{G}^{N}\left(v_{1}, v_{5}\right)=$ $-0.8, \quad \operatorname{CONN}_{G}^{P}\left(v_{2}, v_{4}\right)=0.4, \quad \operatorname{CONN}_{G}^{N}\left(v_{2}, v_{4}\right)=-0.9$, $\operatorname{CONN}_{G}^{P}\left(v_{2}, v_{5}\right)=0.4, \operatorname{CONN}_{G}^{N}\left(v_{2}, v_{5}\right)=-0.9, \operatorname{CONN}_{G}^{P}$ $\left(v_{3}, v_{4}\right)=0.8, \operatorname{CONN}_{G}^{N}\left(v_{2}, v_{5}\right)=-0.8, \quad \operatorname{CONN}_{G}^{P}\left(v_{3}, v_{5}\right)=$ $0.8, \mathrm{CONN}_{G}^{N}\left(v_{3}, v_{5}\right)=-0.8, \mathrm{CONN}_{G}^{P}\left(v_{4}, v_{5}\right)=0.8, \mathrm{CONN}_{G}^{N}$ $\left(v_{4}, v_{5}\right)=-0.9, \quad C_{G}\left(v_{1}\right)=(0.425,-0.5), \quad C S_{G}\left(v_{2}\right)=$ $(0.425,-0.775), C S_{G}\left(v_{3}\right)=(0.6,-0.725), C S_{G}\left(v_{4}\right)=(0.6$, $-0.775), \quad C S_{G}\left(v_{5}\right)=(0.6,-0.775), \quad$ and $\quad C S(G)=$ $(0.53,-0.71)$.

Furthermore, we get $\operatorname{CONN}_{G-\left\{v_{1}\right\}}^{P}\left(v_{2}, v_{3}\right)=0.3$, $\operatorname{CONN}_{G-\left\{v_{1}\right\}}^{N}\left(v_{2}, v_{3}\right)=-0.8, \quad \operatorname{CONN}_{G-\left\{v_{1}\right\}}^{P-\left\{v_{1}\right\}}\left(v_{2}, v_{4}\right)=0.3$,

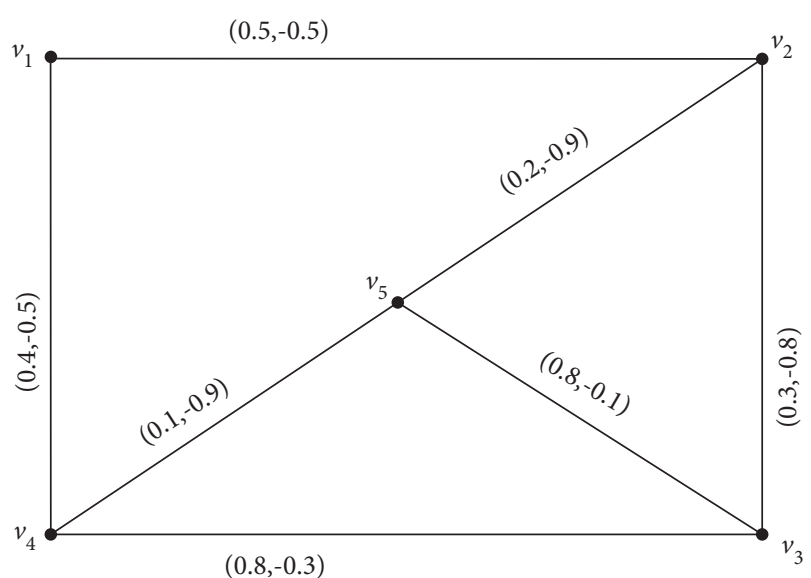

Figure 3: A bipolar fuzzy graph.

$\operatorname{CONN}_{G-\left\{v_{1}\right\}}^{N} \quad\left(v_{2}, v_{4}\right)=-0.9, \quad \operatorname{CONN}_{G-\left\{v_{1}\right\}}^{P}\left(v_{2}, v_{5}\right)=0.3$, $\operatorname{CONN}_{G-\left\{v_{1}\right\}}^{N}\left(v_{2}, v_{5}\right)=-0.9, \quad \operatorname{CONN}_{G-\left\{v_{1}\right\}}^{P} \quad\left(v_{3}, v_{4}\right)=0.8$, $\operatorname{CONN}_{G-\left\{v_{1}\right\}}^{N}\left(v_{3}, v_{4}\right)=-0.8, \quad \operatorname{CONN}_{G-\left\{v_{1}\right\}}^{P} \quad\left(v_{3}, v_{5}\right)=0.8$, $\mathrm{CONN}_{G-\left\{v_{1}\right\}}^{N} \quad\left(v_{3}, v_{5}\right)=-0.8, \quad \operatorname{CONN}_{G-\left\{v_{1}\right\}}^{P}\left(v_{4}, v_{5}\right)=0.8$, $\operatorname{CONN}_{G-\left\{v_{1}\right\}}^{N}\left(v_{4}, v_{5}\right)=-0.9, C S_{G-\left\{v_{1}\right\}}\left(v_{2}\right)=(0.3,-(13 / 15))$, $C S_{G-\left\{v_{1}\right\}}\left(v_{3}\right)=((19 / 30),-0.8), \quad C S_{G-\left\{v_{1}\right\}}\left(v_{4}\right)=\quad((19 / 30)$, $-(13 / 15)), \quad C S_{G-\left\{v_{1}\right\}}\left(v_{5}\right)=((19 / 30),-(13 / 15)), \quad C S(G-$ $\left.\left\{v_{1}\right\}\right)=(0.55,-0.85)$.

$\operatorname{CONN}_{G-\left\{v_{2}\right\}}^{P}\left(v_{1}, v_{3}\right)=0.4, \mathrm{CONN}_{G-\left\{v_{2}\right\}}^{N}\left(v_{1}, v_{3}\right)=-0.3$, $\operatorname{CONN}_{G-\left\{v_{2}\right\}}^{P}\left(v_{1}, v_{4}\right)=0.4, \operatorname{CONN}_{G-\left\{v_{2}\right\}}^{N}\left(v_{1}, v_{4}\right)=-0.5$, $\operatorname{CONN}_{G-\left\{v_{2}\right\}}^{P} \quad\left(v_{1}, v_{5}\right)=0.4, \operatorname{CONN}_{G-\left\{v_{2}\right\}}^{N} \quad\left(v_{1}, v_{5}\right)=-0.5$, $\operatorname{CONN}_{G-\left\{v_{2}\right\}}^{P}\left(v_{3}, v_{4}\right)=0.8, \quad \operatorname{CONN}_{G-\left\{v_{2}\right\}}^{N} \quad\left(v_{3}, v_{4}\right)=-0.3$, $\operatorname{CONN}_{G-\left\{v_{2}\right\}}^{P} \quad\left(v_{3}, v_{5}\right)=0.8, \quad \operatorname{CONN}_{G-\left\{v_{2}\right\}}^{N}\left(v_{3}, v_{5}\right)=-0.3$, $\operatorname{CONN}_{G-\left\{v_{2}\right\}}^{P}\left(v_{4}, v_{5}\right)=0.8, \quad \operatorname{CONN}_{G-\left\{v_{2}\right\}}^{N} \quad\left(v_{4}, v_{5}\right)=-0.9$, $C S_{G-\left\{v_{2}\right\}}\left(v_{1}\right)=(0.4,-(13 / 30)), C S_{G-\left\{v_{2}\right\}}\left(v_{3}\right)=((2 / 3),-0.3)$, $C S_{G-\left\{v_{2}\right\}}\left(v_{4}\right)=((2 / 3),-(17 / 30)), \quad C S_{G-\left\{v_{2}\right\}}\left(v_{5}\right)=((2 / 3)$, $-(17 / 30))$, and $C S\left(G-\left\{v_{2}\right\}\right)=(0.6,-(7 / 15))$.

$\operatorname{CONN}_{G-\left\{v_{3}\right\}}^{P}\left(v_{1}, v_{2}\right)=0.5, \operatorname{CONN}_{G-\left\{v_{3}\right\}}^{N}\left(v_{1}, v_{2}\right)=-0.5$, $\operatorname{CONN}_{G-\left\{v_{3}\right\}}^{P}\left(v_{1}, v_{4}\right)=0.4, \quad \operatorname{CONN}_{G-\left\{v_{3}\right\}}^{N}\left(v_{1}, v_{4}\right)=-0.5$, $\operatorname{CONN}_{G-\left\{v_{3}\right\}}^{P} \quad\left(v_{1}, v_{5}\right)=0.2, \quad \operatorname{CONN}_{G-\left\{v_{3}\right\}}^{N}\left(v_{1}, v_{5}\right)=-0.5$, $\operatorname{CONN}_{G-\left\{v_{3}\right\}}^{P}\left(v_{2}, v_{4}\right)=0.4, \quad \operatorname{CONN}_{G-\left\{v_{3}\right\}}^{N} \quad\left(v_{2}, v_{4}\right)=-0.9$, $\operatorname{CONN}_{G-\left\{v_{3}\right\}}^{P} \quad\left(v_{2}, v_{5}\right)=0.2, \quad \operatorname{CONN}_{G-\left\{v_{3}\right\}}^{N}\left(v_{2}, v_{5}\right)=-0.9$, $\operatorname{CONN}_{G-\left\{v_{3}\right\}}^{P}\left(v_{4}, v_{5}\right)=0.2, \quad \operatorname{CONN}_{G-\left\{v_{3}\right\}}^{N} \quad\left(v_{4}, v_{5}\right)=-0.9$, $C S_{G-\left\{v_{3}\right\}}\left(v_{1}\right)=((11 / 30),-0.5), \quad C S_{G-\left\{v_{3}\right\}}\left(v_{2}\right)=((11 / 30)$, - (23/30)), $C S_{G-\left\{v_{3}\right\}}\left(v_{4}\right)=((1 / 3),-(23 / 30)), C S_{G-\left\{v_{3}\right\}}\left(v_{5}\right)=$ $(0.2,-(23 / 30))$, and $C S\left(G-\left\{v_{3}\right\}\right)=((19 / 60),-0.7)$.

$\operatorname{CONN}_{G-\left\{v_{4}\right\}}^{P}\left(v_{1}, v_{2}\right)=0.5, \mathrm{CONN}_{G-\left\{v_{4}\right\}}^{N}\left(v_{1}, v_{2}\right)=-0.5$, $\operatorname{CONN}_{G-\left\{v_{4}\right\}}^{P}\left(v_{1}, v_{3}\right)=0.3, \quad \operatorname{CONN}_{G-\left\{v_{4}\right\}}^{N}\left(v_{1}, v_{3}\right)=-0.5$, $\operatorname{CONN}_{G-\left\{v_{4}\right\}}^{P} \quad\left(v_{1}, v_{5}\right)=0.3, \operatorname{CONN}_{G-\left\{v_{4}\right\}}^{N} \quad\left(v_{1}, v_{5}\right)=-0.5$, $\operatorname{CONN}_{G-\left\{v_{4}\right\}}^{P} \quad\left(v_{2}, v_{3}\right)=0.3, \quad \operatorname{CONN}_{G-\left\{v_{4}\right\}}^{N}\left(v_{2}, v_{3}\right)=-0.8$, $\operatorname{CONN}_{G-\left\{v_{4}\right\}}^{P}\left(v_{2}, v_{5}\right)=0.3, \quad \operatorname{CONN}_{G-\left\{v_{4}\right\}}^{N} \quad\left(v_{2}, v_{5}\right)=-0.9$, $\mathrm{CONN}_{G-\left\{v_{4}\right\}}^{P}\left(v_{3}, v_{5}\right)=0.8, \quad \mathrm{CONN}_{G-\left\{v_{4}\right\}}^{N}\left(v_{3}, v_{5}\right)=-0.8$, 


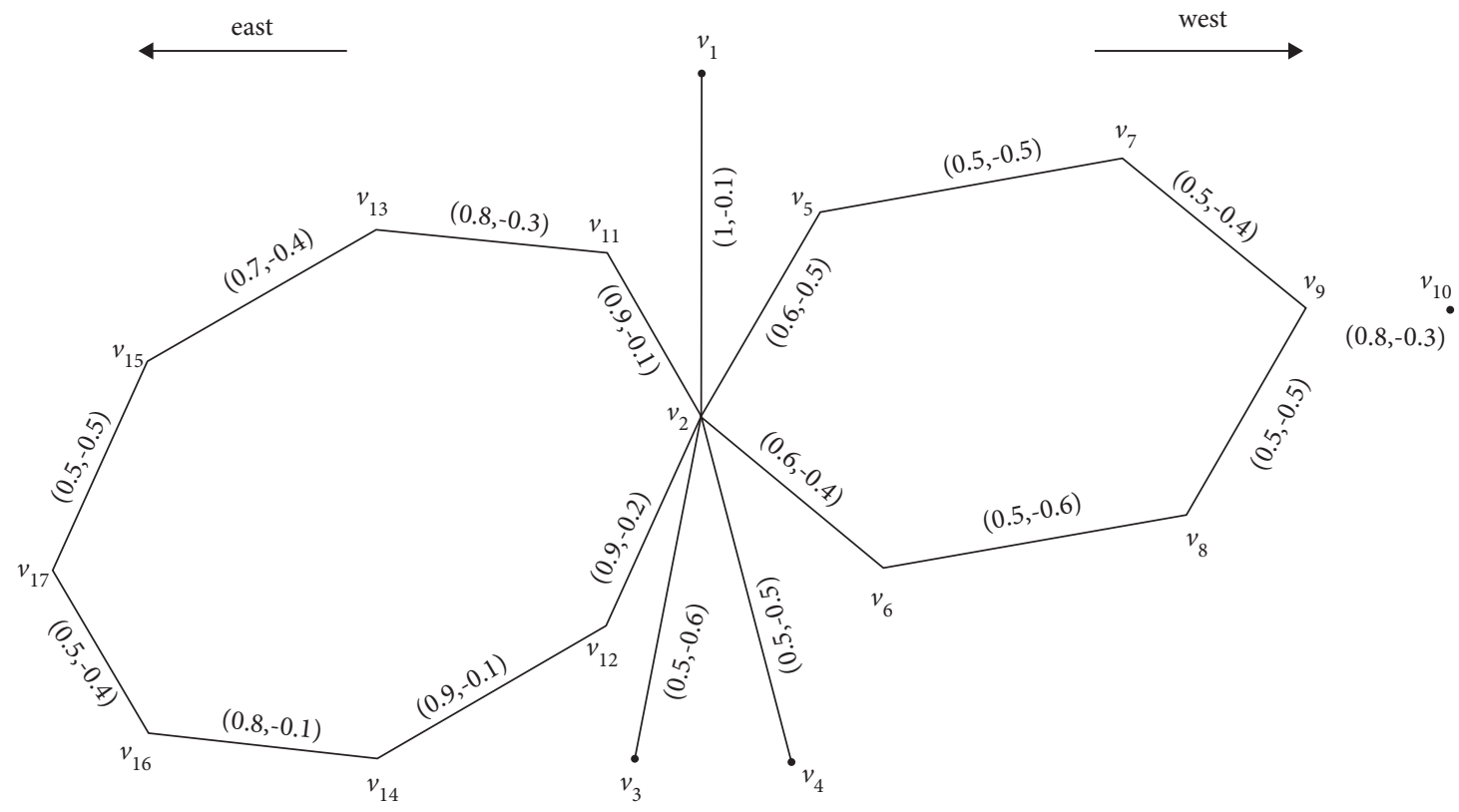

FIgURE 4: Campus topology graph.

$C S_{G-\left\{v_{4}\right\}}\left(v_{1}\right)=((11 / 30),-0.5), \quad C S_{G-\left\{v_{4}\right\}}\left(v_{2}\right)=((11 / 30)$, -(11/15)), $\quad C S_{G-\left\{v_{4}\right\}}\left(v_{3}\right)=((7 / 15),-0.7), \quad C S_{G-\left\{v_{4}\right\}}\left(v_{5}\right)=$ $((7 / 15),-(11 / 15))$, and $C S\left(G-\left\{v_{4}\right\}\right)=((5 / 12),-(2 / 3))$. $\operatorname{CONN}_{G-\left\{v_{5}\right\}}^{P}\left(v_{1}, v_{2}\right)=0.5, \operatorname{CONN}_{G-\left\{v_{5}\right\}}^{N}\left(v_{1}, v_{2}\right)=-0.5$, $\operatorname{CONN}_{G-\left\{v_{5}\right\}}^{P} \quad\left(v_{1}, v_{3}\right)=0.4, \quad \operatorname{CONN}_{G-\left\{v_{5}\right\}}^{\mathcal{N}\left\{v_{5}\right\}}\left(v_{1}, v_{3}\right)=-0.5$, $\operatorname{CONN}_{G-\left\{v_{5}\right\}}^{P}\left(v_{1}, v_{4}\right)=0.4, \quad \operatorname{CONN}_{G-\left\{v_{5}\right\}}^{N} \quad\left(v_{1}, v_{4}\right)=-0.5$, $\operatorname{CONN}_{G-\left\{v_{5}\right\}}^{P} \quad\left(v_{2}, v_{3}\right)=0.4, \quad \operatorname{CONN}_{G-\left\{v_{5}\right\}}^{N}\left(v_{2}, v_{3}\right)=-0.8$, $\operatorname{CONN}_{G-\left\{v_{5}\right\}}^{P}\left(v_{2}, v_{4}\right)=0.4, \quad \operatorname{CONN}_{G-\left\{v_{5}\right\}}^{N} \quad\left(v_{2}, v_{4}\right)=-0.5$, $\operatorname{CONN}_{G-\left\{v_{5}\right\}}^{P} \quad\left(v_{3}, v_{4}\right)=0.8, \operatorname{CONN}_{G-\left\{v_{5}\right\}}^{N} \quad\left(v_{3}, v_{4}\right)=-0.5$, $C S_{G-\left\{v_{5}\right\}}\left(v_{1}\right)=((13 / 30),-0.5), \quad C S_{G-\left\{v_{5}\right\}}\left(v_{2}\right)=((13 / 30)$, -0.6), $C S_{G-\left\{v_{5}\right\}}\left(v_{3}\right)=((8 / 15),-0.6), C S_{G-\left\{v_{5}\right\}}\left(v_{4}\right)=((8 / 15)$, $-0.5)$, and $C S\left(G-\left\{v_{5}\right\}\right)=((29 / 60),-0.55)$.

Note that $C S^{P}\left(G-\left\{v_{3}\right\}\right)<C S^{P}(G)$ and $C S^{N}\left(G-\left\{v_{3}\right\}\right)>C S^{N}(G) ; \quad C S^{P}\left(G-\left\{v_{4}\right\}\right)<C S^{P}(G) \quad$ and $C S^{N}\left(G-\left\{v_{4}\right\}\right)>C S^{N}(G) ; \quad C S^{P}\left(G-\left\{v_{5}\right\}\right)<C S^{P}(G)$ and $C S^{N}\left(G-\left\{v_{5}\right\}\right)>C S^{N}(G)$; hence $v_{3}, v_{4}$, and $v_{5}$ are bipolar connectivity status-reducing vertices. By $\operatorname{CS}^{P}\left(G-\left\{v_{1}\right\}\right)>$ $C S^{P}(G)$ and $C S^{N}\left(G-\left\{v_{1}\right\}\right)<C S^{N}(G)$, we infer that $v_{1}$ is a bipolar connectivity status-enhancing vertex. However, $v_{2}$ does not belong to any type of vertex.

\section{Campus Network Bandwidth Allocation Application}

The information management office of each university is responsible for the distribution and scheduling of its network resources, including the purchase of bandwidth, which is allocated to various colleges and common institutions such as libraries, gymnasium, school hospital, and cafeterias. According to the geographic location of each institution in the school, the network topology is shown in Figure 4, and
TABle 1: Correspondence between vertices and institutions in campus.

\begin{tabular}{lc}
\hline Vertex & The corresponding institutions \\
\hline$v_{1}$ & Library \\
$v_{2}$ & Administration building \\
$v_{3}$ & Stadium \\
$v_{4}$ & Logistics office and university hospital \\
$v_{5}$ & School of Art \\
$v_{6}$ & School of Foreign Languages and Literature \\
$v_{7}$ & School of Economics and Management \\
$v_{8}$ & School of History and Administration \\
$v_{9}$ & Student activity center \\
$v_{10}$ & Student apartments and cafeterias (west area) \\
$v_{11}$ & School of Physics and Electronic Information \\
$v_{12}$ & School of Chemical Engineering and Materials \\
$v_{13}$ & School of Life Science \\
$v_{14}$ & School of Energy and Environment Science \\
$v_{15}$ & School of Mathematics and Computer Science \\
$v_{16}$ & School of Geography \\
$v_{17}$ & Student apartments and cafeterias (east area) \\
\hline
\end{tabular}

the institution corresponding to each vertex is shown in Table 1.

It can be seen from the topological representation graph that the entire university is divided into two areas: the east area and the west area, centered on the library and the administrative building. The west area is dominated by the schools of humanities and arts, and the east area is dominated by the departments of science and engineering. There are student dormitories and canteens on both sides. It should be noted that this is a simplified topology graph. In the actual campus structure, there are also parks, teaching buildings, lakes, and so on in the circles enclosed by the east and west district schools, which we does not show these facilities all in Figure 3. 
TABLE 2: Computing the values of connectivities for each pair of vertices.

\begin{tabular}{|c|c|c|c|}
\hline Vertex pair & $\left(\mathrm{CONN}_{G}^{P}, \mathrm{CONN}_{G}^{N}\right)$ & Vertex pair & $\left(\mathrm{CONN}_{G}^{P}, \mathrm{CONN}_{G}^{N}\right)$ \\
\hline$\left(v_{1}, v_{2}\right)$ & $(1,-0.1)$ & $\left(v_{1}, v_{3}\right)$ & $(0.5,-0.1)$ \\
\hline$\left(v_{1}, v_{4}\right)$ & $(0.5,-0.1)$ & $\left(v_{1}, v_{5}\right)$ & $(0.6,-0.1)$ \\
\hline$\left(v_{1}, v_{6}\right)$ & $(0.6,-0.1)$ & $\left(v_{1}, v_{7}\right)$ & $(0.5,-0.1)$ \\
\hline$\left(v_{1}, v_{8}\right)$ & $(0.5,-0.1)$ & $\left(v_{1}, v_{9}\right)$ & $(0.5,-0.1)$ \\
\hline$\left(v_{1}, v_{10}\right)$ & $(0.5,-0.1)$ & $\left(v_{1}, v_{11}\right)$ & $(0.9,-0.1)$ \\
\hline$\left(v_{1}, v_{12}\right)$ & $(0.9,-0.1)$ & $\left(v_{1}, v_{13}\right)$ & $(0.8,-0.1)$ \\
\hline$\left(v_{1}, v_{14}\right)$ & $(0.9,-0.1)$ & $\left(v_{1}, v_{15}\right)$ & $(0.7,-0.1)$ \\
\hline$\left(v_{1}, v_{16}\right)$ & $(0.8,-0.1)$ & $\left(v_{1}, v_{17}\right)$ & $(0.5,-0.1)$ \\
\hline$\left(v_{2}, v_{3}\right)$ & $(0.5,-0.6)$ & $\left(v_{2}, v_{4}\right)$ & $(0.5,-0.5)$ \\
\hline$\left(v_{2}, v_{5}\right)$ & $(0.6,-0.5)$ & $\left(v_{2}, v_{6}\right)$ & $(0.6,-0.4)$ \\
\hline$\left(v_{2}, v_{7}\right)$ & $(0.5,-0.5)$ & $\left(v_{2}, v_{8}\right)$ & $(0.5,-0.4)$ \\
\hline$\left(v_{2}, v_{9}\right)$ & $(0.5,-0.4)$ & $\left(v_{2}, v_{10}\right)$ & $(0.5,-0.3)$ \\
\hline$\left(v_{2}, v_{11}\right)$ & $(0.9,-0.1)$ & $\left(v_{2}, v_{12}\right)$ & $(0.9,-0.2)$ \\
\hline$\left(v_{2}, v_{13}\right)$ & $(0.8,-0.1)$ & $\left(v_{2}, v_{14}\right)$ & $(0.9,-0.1)$ \\
\hline$\left(v_{2}, v_{15}\right)$ & $(0.7,-0.1)$ & $\left(v_{2}, v_{16}\right)$ & $(0.8,-0.1)$ \\
\hline$\left(v_{2}, v_{17}\right)$ & $(0.5,-0.1)$ & $\left(v_{3}, v_{4}\right)$ & $(0.5,-0.5)$ \\
\hline$\left(v_{3}, v_{5}\right)$ & $(0.5,-0.5)$ & $\left(v_{3}, v_{6}\right)$ & $(0.5,-0.4)$ \\
\hline$\left(v_{3}, v_{7}\right)$ & $(0.5,-0.5)$ & $\left(v_{3}, v_{8}\right)$ & $(0.5,-0.4)$ \\
\hline$\left(v_{3}, v_{9}\right)$ & $(0.5,-0.4)$ & $\left(v_{3}, v_{10}\right)$ & $(0.5,-0.3)$ \\
\hline$\left(v_{3}, v_{11}\right)$ & $(0.5,-0.1)$ & $\left(v_{3}, v_{12}\right)$ & $(0.5,-0.2)$ \\
\hline$\left(v_{3}, v_{13}\right)$ & $(0.5,-0.1)$ & $\left(v_{3}, v_{14}\right)$ & $(0.5,-0.1)$ \\
\hline$\left(v_{3}, v_{15}\right)$ & $(0.5,-0.1)$ & $\left(v_{3}, v_{16}\right)$ & $(0.5,-0.1)$ \\
\hline$\left(v_{3}, v_{17}\right)$ & $(0.5,-0.1)$ & $\left(v_{4}, v_{5}\right)$ & $(0.5,-0.5)$ \\
\hline$\left(v_{4}, v_{6}\right)$ & $(0.5,-0.4)$ & $\left(v_{4}, v_{7}\right)$ & $(0.5,-0.5)$ \\
\hline$\left(v_{4}, v_{8}\right)$ & $(0.5,-0.4)$ & $\left(v_{4}, v_{9}\right)$ & $(0.5,-0.4)$ \\
\hline$\left(v_{4}, v_{10}\right)$ & $(0.5,-0.3)$ & $\left(v_{4}, v_{11}\right)$ & $(0.5,-0.1)$ \\
\hline$\left(v_{4}, v_{12}\right)$ & $(0.5,-0.2)$ & $\left(v_{4}, v_{13}\right)$ & $(0.5,-0.1)$ \\
\hline$\left(v_{4}, v_{14}\right)$ & $(0.5,-0.1)$ & $\left(v_{4}, v_{15}\right)$ & $(0.5,-0.1)$ \\
\hline$\left(v_{4}, v_{16}\right)$ & $(0.5,-0.1)$ & $\left(v_{4}, v_{17}\right)$ & $(0.5,-0.1)$ \\
\hline$\left(v_{5}, v_{6}\right)$ & $(0.6,-0.4)$ & $\left(v_{5}, v_{7}\right)$ & $(0.5,-0.5)$ \\
\hline$\left(v_{5}, v_{8}\right)$ & $(0.5,-0.4)$ & $\left(v_{5}, v_{9}\right)$ & $(0.5,-0.4)$ \\
\hline$\left(v_{5}, v_{10}\right)$ & $(0.5,-0.3)$ & $\left(v_{5}, v_{11}\right)$ & $(0.6,-0.1)$ \\
\hline$\left(v_{5}, v_{12}\right)$ & $(0.6,-0.2)$ & $\left(v_{5}, v_{13}\right)$ & $(0.6,-0.1)$ \\
\hline$\left(v_{5}, v_{14}\right)$ & $(0.6,-0.1)$ & $\left(v_{5}, v_{15}\right)$ & $(0.6,-0.1)$ \\
\hline$\left(v_{5}, v_{16}\right)$ & $(0.6,-0.1)$ & $\left(v_{5}, v_{17}\right)$ & $(0.5,-0.1)$ \\
\hline$\left(v_{6}, v_{7}\right)$ & $(0.5,-0.4)$ & $\left(v_{6}, v_{8}\right)$ & $(0.5,-0.6)$ \\
\hline$\left(v_{6}, v_{9}\right)$ & $(0.5,-0.5)$ & $\left(v_{6}, v_{10}\right)$ & $(0.5,-0.3)$ \\
\hline$\left(v_{6}, v_{11}\right)$ & $(0.6,-0.1)$ & $\left(v_{6}, v_{12}\right)$ & $(0.6,-0.2)$ \\
\hline$\left(v_{6}, v_{13}\right)$ & $(0.6,-0.1)$ & $\left(v_{6}, v_{14}\right)$ & $(0.6,-0.1)$ \\
\hline$\left(v_{6}, v_{15}\right)$ & $(0.6,-0.1)$ & $\left(v_{6}, v_{16}\right)$ & $(0.6,-0.1)$ \\
\hline$\left(v_{6}, v_{17}\right)$ & $(0.5,-0.1)$ & $\left(v_{7}, v_{8}\right)$ & $(0.5,-0.4)$ \\
\hline$\left(v_{7}, v_{9}\right)$ & $(0.5,-0.4)$ & $\left(v_{7}, v_{10}\right)$ & $(0.5,-0.3)$ \\
\hline$\left(v_{7}, v_{11}\right)$ & $(0.5,-0.1)$ & $\left(v_{7}, v_{12}\right)$ & $(0.5,-0.2)$ \\
\hline$\left(v_{7}, v_{13}\right)$ & $(0.5,-0.1)$ & $\left(v_{7}, v_{14}\right)$ & $(0.5,-0.1)$ \\
\hline$\left(v_{7}, v_{15}\right)$ & $(0.5,-0.1)$ & $\left(v_{7}, v_{16}\right)$ & $(0.5,-0.1)$ \\
\hline$\left(v_{7}, v_{17}\right)$ & $(0.5,-0.1)$ & $\left(v_{8}, v_{9}\right)$ & $(0.5,-0.5)$ \\
\hline$\left(v_{8}, v_{10}\right)$ & $(0.5,-0.3)$ & $\left(v_{8}, v_{11}\right)$ & $(0.5,-0.1)$ \\
\hline$\left(v_{8}, v_{12}\right)$ & $(0.5,-0.2)$ & $\left(v_{8}, v_{13}\right)$ & $(0.5,-0.1)$ \\
\hline$\left(v_{8}, v_{14}\right)$ & $(0.5,-0.1)$ & $\left(v_{8}, v_{15}\right)$ & $(0.5,-0.1)$ \\
\hline$\left(v_{8}, v_{16}\right)$ & $(0.5,-0.1)$ & $\left(v_{8}, v_{17}\right)$ & $(0.5,-0.1)$ \\
\hline$\left(v_{9}, v_{10}\right)$ & $(0.8,-0.3)$ & $\left(v_{9}, v_{11}\right)$ & $(0.5,-0.1)$ \\
\hline$\left(v_{9}, v_{12}\right)$ & $(0.5,-0.2)$ & $\left(v_{9}, v_{13}\right)$ & $(0.5,-0.1)$ \\
\hline$\left(v_{9}, v_{14}\right)$ & $(0.5,-0.1)$ & $\left(v_{9}, v_{15}\right)$ & $(0.5,-0.1)$ \\
\hline$\left(v_{9}, v_{16}\right)$ & $(0.5,-0.1)$ & $\left(v_{9}, v_{17}\right)$ & $(0.5,-0.1)$ \\
\hline$\left(v_{10}, v_{11}\right)$ & $(0.5,-0.1)$ & $\left(v_{10}, v_{12}\right)$ & $(0.5,-0.2)$ \\
\hline$\left(v_{10}, v_{13}\right)$ & $(0.5,-0.1)$ & $\left(v_{10}, v_{14}\right)$ & $(0.5,-0.1)$ \\
\hline$\left(v_{10}, v_{15}\right)$ & $(0.5,-0.1)$ & $\left(v_{10}, v_{16}\right)$ & $(0.5,-0.1)$ \\
\hline$\left(v_{10}, v_{17}\right)$ & $(0.5,-0.1)$ & $\left(v_{11}, v_{12}\right)$ & $(0.9,-0.1)$ \\
\hline$\left(v_{11}, v_{13}\right)$ & $(0.8,-0.3)$ & $\left(v_{11}, v_{14}\right)$ & $(0.9,-0.1)$ \\
\hline$\left(v_{11}, v_{15}\right)$ & $(0.7,-0.3)$ & $\left(v_{11}, v_{16}\right)$ & $(0.8,-0.3)$ \\
\hline
\end{tabular}


TABLE 2: Continued.

\begin{tabular}{lccr}
\hline Vertex pair & $\left(\mathrm{CONN}_{G}^{P}, \mathrm{CONN}_{G}^{N}\right)$ & Vertex pair & $\left(\mathrm{CONN}_{G}^{P}, \mathrm{CONN}_{G}^{N}\right)$ \\
\hline$\left(v_{11}, v_{17}\right)$ & $(0.5,-0.3)$ & $\left(v_{12}, v_{13}\right)$ & $(0.8,-0.1)$ \\
$\left(v_{12}, v_{14}\right)$ & $(0.9,-0.1)$ & $\left(v_{12}, v_{15}\right)$ & $(0.7,-0.1)$ \\
$\left(v_{12}, v_{16}\right)$ & $(0.8,-0.1)$ & $\left(v_{12}, v_{17}\right)$ & $(0.5,-0.1)$ \\
$\left(v_{13}, v_{14}\right)$ & $(0.8,-0.1)$ & $\left(v_{13}, v_{15}\right)$ & $(0.7,-0.4)$ \\
$\left(v_{13}, v_{16}\right)$ & $(0.8,-0.4)$ & $\left(v_{13}, v_{17}\right)$ & $(0.5,-0.4)$ \\
$\left(v_{14}, v_{15}\right)$ & $(0.7,-0.1)$ & $\left(v_{14}, v_{16}\right)$ & $(0.8,-0.1)$ \\
$\left(v_{14}, v_{17}\right)$ & $(0.5,-0.1)$ & $\left(v_{15}, v_{16}\right)$ & $(0.7,-0.4)$ \\
$\left(v_{15}, v_{17}\right)$ & $(0.5,-0.5)$ & $\left(v_{16}, v_{17}\right)$ & $(0.5,-0.4)$ \\
\hline
\end{tabular}

TABLE 3: Calculation the connectivity status of vertices.

\begin{tabular}{lccccc}
\hline Vertex & $\left(C S_{G}^{P}(v), C S_{G}^{N}(v)\right)$ & Vertex & $\left(C S_{G}^{P}(v), C S_{G}^{N}(v)\right)$ & Vertex & $\left(C S_{G}^{P}(v), C S_{G}^{N}(v)\right)$ \\
\hline$v_{1}$ & $((107 / 160),-0.1)$ & $v_{2}$ & $((107 / 160),-(9 / 32))$ & $v_{3}$ & $(0.5,-(9 / 32))$ \\
$v_{4}$ & $(0.5,-(11 / 40))$ & $v_{5}$ & $((11 / 20),-(11 / 40))$ & $v_{6}$ & $((89 / 160),-(43 / 160))$ \\
$v_{7}$ & $(0.5,-(11 / 40))$ & $v_{8}$ & $(0.5,-(43 / 160))$ & $v_{9}$ & $((83 / 160),-(21 / 80))$ \\
$v_{10}$ & $((83 / 160),-(33 / 160))$ & $v_{11}$ & $((53 / 80),-(3 / 20))$ & $v_{12}$ & $((53 / 80),-(5 / 32))$ \\
$v_{13}$ & $((51 / 80),-(27 / 160))$ & $v_{14}$ & $((53 / 80),-0.1)$ & $v_{15}$ & $(0.6,-(13 / 80))$ \\
$v_{16}$ & $((51 / 80),-(27 / 160))$ & $v_{17}$ & $(0.5,-(7 / 40))$ & & \\
\hline
\end{tabular}

Assume that $\mu_{A}^{P}(v)=1$ and $\mu_{A}^{N}(v)=-1$ for all vertices in this example. Considering the network cable layout of the channel between the vertices, router settings, uplink capacity and downlink capacity, link delay time, arrangement time, and other positive and negative elements, the membership function values of each edge are shown in Figure 4. The connectivities of all pairs of vertices are listed in Table 2.

In light of Table 2 , the connectivity status of vertices is determined in Table 3.

For the bandwidth allocation problem, the average minimum connectivity in the entire network is required so that the connectivity of each vertex is not lower than this minimum threshold. Assuming that the vertex where the college is located also represents the router set by the department, the edge weight represents the corresponding route and the threshold is set to $(0.5,-9 / 32)$. That is to say, it requires $C S_{G}^{P}(v)>0.5$ and $C S_{G}^{N}(v)>-9 / 32$. Note that the vertices $v_{3}, v_{4}, v_{7}$, and $v_{17}$ do not satisfy $C S_{G}^{P}(v)>0.5$, and the vertices $v_{2}$ and $v_{3}$ do not satisfying $C S_{G}^{N}(v)>-9 / 32$. Therefore, we must focus on these three vertices to enhance their connected state value to achieve the minimum connection constraint. The connected state analysis of the vertices can be used to obtain the correct direction for designing a balanced network.

\section{Conclusion}

In computer networks, there are many negative uncertain factors, such as the delay of the network apex, the capacity limitation of the uplink and the downlink, the status of the queuing sequence, and so on. Since bipolar fuzzy graphs have negative membership functions that specifically describe these negative uncertain factors, bipolar fuzzy graphs are more flexible in specific network applications than general fuzzy graphs.
In this paper, we introduce the connectivity status in the bipolar fuzzy graph which reflects the positive and negative connectedness in a connected network for each vertex, as well as the whole network. Its main function is to help us analyze the connectivity strength of each vertex in the bipolar network and find the weakest link in the entire network through the comparison between the vertices to identify security risks, strengthen network disadvantages in time, and prevent possible attacks against network weaknesses.

The following contents can be a topic for continued research:

(1) The connectivity status should be further considered in special bipolar graph settings, for instance, bipolar fuzzy incidence graph, bipolar intuitionistic fuzzy graph, and bipolar Pythagoras fuzzy graph

(2) The bipolar connectivity status in specific graph structures should be discussed, such as bipolar planar graph and bipolar cube graph

\section{Data Availability}

All experiment data are included in this article.

\section{Conflicts of Interest}

The authors declare that there are no conflicts of interest in publishing this paper.

\section{Acknowledgments}

This work was supported by Guangdong Basic and Applied Basic Research Foundation of China (No. 2021A1515110834), Natural Science Foundation of China 
(No. 51574232), Guangdong Natural Science Foundation of China (No. 2020A1515010784), and Guangdong University of Science and Technology University Major Scientific Research Achievement Cultivation Program Project (No. GKY-2020CQPY-2).

\section{References}

[1] K. F. Cheung and M. G. H. Bell, "Improving connectivity of compromised digital networks via algebraic connectivity maximisation," European Journal of Operational Research, vol. 294, no. 1, pp. 353-364, 2021.

[2] M. Yemini, A. Somekh-Baruch, R. Cohen, and A. Leshem, "The simultaneous connectivity of cognitive networks," IEEE Transactions on Information Theory, vol. 65, no. 11, pp. 6911-6930, 2019.

[3] F. Burla, S. Dussi, C. M. Torres, J. Tauber, J. V. D. Gucht, and G. H. Koenderink, "Connectivity and plasticity determine collagen network fracture," Proceedings of the National Academy of Sciences, vol. 117, no. 15, pp. 8326-8334, 2020.

[4] C. D. Denison, M. C. Scott, K. M. Kubach, and B. K. Peoples, "Incorporating network connectivity into stream classification frameworks," Environmental Management, vol. 67, no. 2, pp. 291-307, 2021.

[5] T. A. Hummer, M. G. Yung, J. Goñi et al., "Functional network connectivity in early-stage schizophrenia," Schizophrenia Research, vol. 218, pp. 107-115, 2020.

[6] J. Ma, Q. Li, and X. Zhou, "Fuzzy edge connectivity and fuzzy local edge connectivity with applications to communication networks," Fuzzy Sets and Systems, vol. 410, pp. 109-125, 2021.

[7] S. Gong, G. Hua, and W. Gao, "Domination of bipolar fuzzy graphs in various settings," International Journal of Computational Intelligence Systems, vol. 14, 2021, https://link. springer.com/article/10.1007/s44196-021-00011-2, Article ID 162.

[8] W. Gao, Y. Chen, and Y. Wang, "Network vulnerability parameter and results on two surfaces," International Journal of Intelligent Systems, vol. 36, no. 8, pp. 4392-4414, 2021.

[9] W. Gao, W. Wang, and Y. Chen, "Tight bounds for the existence of path factors in network vulnerability parameter settings," International Journal of Intelligent Systems, vol. 36, no. 3, pp. 1133-1158, 2021.

[10] R. Kumar, T. Amgoth, and D. Das, "Obstacle-aware connectivity establishment in wireless sensor networks," IEEE Sensors Journal, vol. 21, no. 4, pp. 5543-5552, 2021.

[11] M. Akram, J. M. Dar, and S. Naz, "Pythagorean Dombi fuzzy graphs," Complex \& Intelligent Systems, vol. 6, no. 1, pp. 29-54, 2020.

[12] M. Atef, A. E. F. El Atik, and A. Nawar, "Fuzzy topological structures via fuzzy graphs and their applications," Soft Computing, vol. 25, no. 8, pp. 6013-6027, 2021.

[13] T. Mahapatra, G. Ghorai, and M. Pal, "Fuzzy fractional coloring of fuzzy graph with its application," Journal of Ambient Intelligence and Humanized Computing, vol. 11, no. 11, pp. 5771-5784, 2020.

[14] A. A. Talebi, H. Rashmanlou, and S. H. Sadati, "Intervalvalued intuitionistic fuzzy competition graph," Journal of Multiple-Valued Logic and Soft Computing, vol. 34, no. 3-4, pp. 335-364, 2020.

[15] S. Gong and G. Hua, "Remarks on Wiener index of bipolar fuzzy incidence graphs," Frontiers in Physics, vol. 9, 2021.

[16] M. Binu, S. Mathew, and J. N. Mordeson, "Cyclic connectivity index of fuzzy graphs," IEEE Transactions on Fuzzy Systems, vol. 29, no. 6, pp. 1340-1349, 2021.
[17] S. Amanathulla, B. Bera, and M. Pal, "Balanced picture fuzzy graph with application," Artificial Intelligence Review, vol. 54, no. 7, pp. 5255-5281, 2021.

[18] S. Kalathian, S. Ramalingam, N. Srinivasan, S. Raman, and S. Broumi, "Embedding of fuzzy graphs on topological surfaces," Neural Computing \& Applications, vol. 32, no. 9, pp. 5059-5069, 2020.

[19] M. Akram and A. Sattar, "Competition graphs under complex Pythagorean fuzzy information," Journal of Applied Mathematics and Computing, vol. 63, no. 1-2, pp. 543-583, 2020.

[20] R. Mahapatra, S. Samanta, and M. Pal, "Applications of edge colouring of fuzzy graphs," Informatica, vol. 31, no. 2, pp. 313-330, 2020.

[21] T. Mahmood and U. Ur Rehman, "A novel approach towards bipolar complex fuzzy sets and their applications in generalized similarity measures," International Journal of Intelligent Systems, vol. 37, no. 1, pp. 535-567, 2021.

[22] C. Jana, "Multiple attribute group decision-making method based on extended bipolar fuzzy MABAC approach," Computational and Applied Mathematics, vol. 40, no. 6, 227 pages, 2021.

[23] S. Poulik, S. Das, and G. Ghorai, "Randic index of bipolar fuzzy graphs and its application in network systems," Journal of Applied Mathematics and Computing, 2021.

[24] G. Ali, G. Muhiuddin, A. Adeel, and M. Zain Ul Abidin, "Ranking effectiveness of Covid-19 tests using fuzzy bipolar soft expert sets," Mathematical Problems in Engineering, vol. 2021, Article ID 5874216, 19 pages, 2021.

[25] M. A. Mehmood, M. Akram, M. G. Alharbi, and S. Bashir, "Optimization of LR -type fully bipolar fuzzy linear programming problems," Mathematical Problems in Engineering, vol. 2021, Article ID 1199336, 36 pages, 2021.

[26] G. Özçelik and M. Nalkıran, "An extension of EDAS method equipped with trapezoidal bipolar fuzzy information: an application from healthcare system," International Journal of Fuzzy Systems, vol. 23, no. 7, pp. 2348-2366, 2021.

[27] M. E. Cornejo, D. Lobo, and J. Medina, "On the solvability of bipolar max-product fuzzy relation equations with the standard negation," Fuzzy Sets and Systems, vol. 410, pp. 1-18, 2021.

[28] S. Mathew, J. N. Mordeson, and D. S. Malik, Fuzzy Graph Theory, Springer, Berlin, Germany, 2018.

[29] M. Pal, S. Samanta, and G. Ghorai, Modern Trends in Fuzzy Graph Theory, Springer, Berlin, Germany, 2020.

[30] M. Akram, M. Sarwar, and W. A. Dudek, Graphs for the Analysis of Bipolar Fuzzy Information, Springer, Berlin, Germany, 2021.

[31] S. Chakraverty, S. Tapaswini, and D. Behera, Fuzzy Arbitrary Order System: Fuzzy Fractional Differential Equations and Applications, John Wiley and Sons, Hoboken, NJ, USA, 2016.

[32] S. Chakraverty, Soft Computing in Interdisciplinary Sciences, Springer, Berlin, Germany, 2022.

[33] C. Volosencu and M. A. Aceves-Fernandez, Fuzzy Logic, BoD-Books on Demand, Norderstedt, Germany, 2020.

[34] M. Binu, S. Mathew, and J. N. Mordeson, "Connectivity status of fuzzy graphs," Information Sciences, vol. 573, pp. 382-395, 2021.

[35] S. Poulik and G. Ghorai, "Certain indices of graphs under bipolar fuzzy environment with applications," Soft Computing, vol. 24, no. 7, pp. 5119-5131, 2020.

[36] S. Mathew, H. L. Yang, and J. K. Mathew, "Saturation in fuzzy graphs," New Mathematics and Natural Computation, vol. 14, no. 1, pp. 113-128, 2018. 